\title{
Is there a problem with low energy SN1987A neutrinos?
}

\author{
M L Costantini ${ }^{1,2}$, A Ianni $^{1}$, G Pagliaroli $^{1,2}$, F Vissani $^{1}$ \\ ${ }^{1}$ INFN, Laboratori Nazionali del Gran Sasso, Assergi (AQ), Italy \\ ${ }^{2}$ University of L'Aquila, Coppito (AQ), Italy
}

\begin{abstract}
The observation of several low energy events during SN1987A burst has an important weight in the attempts to learn about the properties of the supernova neutrinos, but is somewhat puzzling if compared with the expectations. In this work, we study the low energy events observed by Kamiokande-II, and consider in particular the possibility that a few of them are due to background. We focus our attention not only on the event 6 , that falls below the energy threshold of $7.5 \mathrm{MeV}$, but also on the other four events that at $1 \sigma$ fall below the energy threshold, namely the events $3,4,10,12$. The a priori expectations on the number of background events above threshold would suggest 1 or 2 background events only. The volume distribution of the Kamiokande-II events is not a uniform distribution at $3 \sigma$, that suggests the presence of background events close or at the border of the volume used for the analysis, including the events $3,4,10$. Next we checked the expected energy distribution assuming that the signal is due to $\bar{\nu}_{e} p \rightarrow e^{+} n$ and that the average antineutrino energy is 14 $\mathrm{MeV}$. The agreement with the observations is not perfect, but it is acceptable at the $11 \%$ confidence level if we include the peak of low energy background events; otherwise, we face a $2.9 \sigma$ problem. The expected energy distribution implies that the evidence for supernova neutrinos is at $10 \sigma$ and that 1-3 background events are plausible. This conclusion does not change strongly when we model the time distribution of the signal, taking into account the presence of an initial luminous phase of neutrino emission. This suggests however that some of the early events (in particular, event 4) are due to supernova neutrinos and not to background. In summary, our comparison between the expectations and the data lead us to formulate the hypothesis that some of the observed low energy events are due to background and that some among them belong to a peculiar phase of emission, that could be further characterized by low energy neutrinos. Such an interpretation is mostly attractive, since it diminishes to a minimum the postulated number of background events and thus improves the agreement between the a priori (model independent) and the a posteriori (model dependent) expectations on the number of background events. We argue on these grounds that there is no significant disagreement between the average energy of the supernova neutrinos seen in Kamiokande-II and the conventional expectations. Alternative possibilities of interpretations are mentioned and briefly discussed.
\end{abstract}




\section{Contents}

1 Motivation and context $\quad 2$

2 Tools to separate signal from background in Kamiokande-II dataset 4

2.1 Spatial distribution . . . . . . . . . . . . . . . . . . . . 6

2.2 Energy distribution . . . . . . . . . . . . . . . . . . . . . . 8

2.2.1 Comparison of the expected and the measured energy distributions . . . . . . . . . . 8

2.2.2 Analysis of the individual events . . . . . . . . . . . . . . . . . . . . . . 9

2.3 Energy threshold . . . . . . . . . . . . . . . . . . . . 11

3 Average energy of SN1987A neutrinos accounting for background $\quad 12$

3.1 A first global approach . . . . . . . . . . . . . . . . . . . . . 13

3.1.1 Model dependent and independent inferences . . . . . . . . . . . . . . . . . . . 14

3.1.2 A tentative explanation of low energy events . . . . . . . . . . . . . . . . . 16

3.2 A second global approach . . . . . . . . . . . . . . . . . . . . . 17

3.2.1 A more refined hypothesis on neutrino luminosity . . . . . . . . . . . . . . 17

3.2.2 Accretion and the meaning of the early events . . . . . . . . . . . . . . 19

3.2.3 Again on the interpretation of low energy events . . . . . . . . . . . . 20

4 Discussion 21

A The assumed background

$\begin{array}{ll}\text { B The assumption of uniform volumetric distribution } & 24\end{array}$

C The Anderson-Darling test

\section{Motivation and context}

On February 23, 1987, several experiments [1, 2, 3, 4] contributed to begin the era of extragalactic neutrino astronomy. These observations had an enormous impact on astrophysics and on particle physics 1 A straightforward interpretation of these data is yet difficult. This is due not only to the fact that we lack a firmly established theory of supernova explosion, but also to certain anomalous features of the data that have been understood, emphasized and analyzed in the course of the time. In particular, this is the case of the average energy deduced by the 12 events observed in Kamiokande-II (KII), which is half of that observed by IMB, and also lower then what expected in theoretical models for supernova neutrino emission, recently reviewed in [14]. The main goal of the present paper is to propose an interpretation of this apparent discrepancy.

\footnotetext{
${ }^{1}$ In order to have an idea of how large was the impact it is sufficient to consider that, on March 2007, there are 172,000 (resp., 3,050) entries on 'SN1987A' in Google (resp., in Google scholar). The SPIRES database lists 578 papers typing the same keyword; our first two references are those with more than 500 citations, whereas those that rank more than 100 citations include theoretical works on extra dimensions, CPT violation, axions, magnetic moments and exotic particles; several studies of the role of neutrino oscillations follow. Finally, the 20th anniversary of this observation was celebrated with conferences held in Moscow (Feb. 20-22, 2007), in Hawaii (Feb. 23-25, 2007) and Venice (Mar. 6-9, 2007) [30.
} 
The low energy feature of KII dataset is clearly reflected in the outcome of two recent analyses of these data, that explore certain possibilities that deviate strongly from theoretical expectations. The first one is by Mirizzi and Raffelt [25, who describe the distribution in $\bar{\nu}_{e}$ energies as $E_{\bar{\nu}_{e}}^{\alpha} \exp \left[-(\alpha+1) E_{\bar{\nu}_{e}} /\langle E\rangle\right]$ and find that the best fit of KII data is provided by $\alpha \sim 0$ (i.e., a monotonically decreasing distribution). The second one is the analysis of Lunardini [26], who adopts a two-component distribution as suggested by three flavor oscillation scenarios and finds that a component with $\left\langle E_{\bar{\nu}_{e}}\right\rangle \sim 5 \mathrm{MeV}$ permits to fit KII data better 2 This type of approaches is useful to emphasize features of the data like the excess of low energy events in KII dataset but one should recall that neither $\left\langle E_{\bar{\nu}_{e}}\right\rangle \sim 5 \mathrm{MeV}$ nor $\alpha \sim 0$ are compatible with the current expectations for supernova neutrino emission, so that in a conservative analysis, these values should not be allowed.

Stated otherwise, there is a trend in modern studies of SN1987A to accept the opinion that KII data are incompatible with the present theoretical expectations and, consequently, to start from this position to investigate more or less radical departures from the conventional paradigm for neutrino emission. One could even think that the failures to obtain a theory of supernova explosion could motivate such an attitude. But the fact that we do not have yet a definitive theory of the explosion does not mean that all theoretical possibilities are a priori equivalent. Furthermore, one should be careful in distinguishing between the problems of getting a theory of supernova explosion and the problem of knowing the distribution of the emitted neutrinos. In the only scenario that has been explored in some details till now, the so called "delayed scenario" [9, 10], a large amount of the neutrinos-up to $90 \%$-is emitted in a phase that follows the explosion: thus, the two problems are to a certain extent independent (we will better analyze the connection in the following). Comparing [14] and [12] we note that the expected range of one crucial parameter, the average $\bar{\nu}_{e}$ energies or temperatures, did not change much since 1989. What changed is the expectation on the temperature of the other antineutrinos and thus the impact of oscillations, that after [14] can be argued to modify only slightly the observable $\bar{\nu}_{e}$ signal. To summarize, the main reasons why we are not convinced that we should abandon the conventional paradigm for neutrino emission are that: 1) the expectations for neutrino emission seem to be stable; 2) conversely, there is no convincing theoretical argument till now supporting an interpretation of the excess of low energy events in terms of low energy supernova neutrinos.

Also, on general statistical ground one should be aware of the risks of using the data-12 events in the case of KII-to infer the characteristics of the model, rather than asking the significance level at which the null-hypothesis (=the theoretical expectation) is ruled out. In principle it is always possible to obtain a perfect fit $\left(\chi^{2}=0\right)$ to the data declaring that the observed distribution coincides with the expected one, but only if one is ready to renounce to the previous knowledge. In short, we believe that a conservative discussion should address other questions: How severe is the deviation from the conventional theoretical expectations? How reliable is the indication of a large amount of low energy supernova neutrinos, inferred from KII observations? Is it possible to conceive other interpretations (more standard than the one proposed in [26] and [25]) of the observed low energy events?

This is why we would like to explore the possibility that some of the low energy events in KII

\footnotetext{
${ }^{2}$ In fact, the question of which is the energy distribution of an 'average' core collapse supernova has important implications for future experiments: e.g., the analysis of SN1987A neutrinos of [26] was used to argue that future search of relic supernova neutrinos could fail because the emitted neutrinos have much lower energy than expected.
} 
are not due to $\bar{\nu}_{e} p \rightarrow e^{+} n$ interactions of supernova neutrinos as usually assumed. The possibility that a few events are due to elastic scattering has been recently reconsidered [23], finding that, although this cannot be excluded, it helps only marginally to explain the excess of low energy events in KII data set. This forced the authors of [23] to admit that a few of these events are of a different origin, and possibly are due to background. This is a conservative position, since it is evident that the KII observations could be polluted by some background events: see figures 4, 5, 10, 11, 12 of the second publication of KII collaboration on SN1987A, i.e. [5]. Furthermore, in Čerenkov type detector it is not possible to know whether a single event is due to signal or to background. In our understanding, the main point of KII collaboration was just that it is not possible that all 12 events are due to background, which means that an observation of supernova neutrinos has been made (as we show in Sect. 2.2, the a priori knowledge of the signal reinforces significantly this evidence). It should be noted that the KII collaboration did not quantify the probability that the 12 events are due to background on individual basis; the only step in this direction was to exclude occasionally one specific event, occurred below the threshold for solar neutrinos events (more discussion on this issue in Sect. 2.3). In this note, we analyze quantitatively the possibility that some background events occurred in the KII dataset, extending and complementing the analysis of [21]. In particular, we focus our attention on the spatial and the energy distribution of the 12 candidate events.

We are aware of the problems of small numbers statistics, that there are many more papers on SN1987A neutrinos than events, and that there is the risk of running into 'forensics' (quoting a witticism of John Beacom). Nevertheless, we should also recall that supernovae are rare events on human timescale, and that all we have at the moment is one observation of supernova neutrinos and some theoretical ideas to compare with. In other words, we feel that we have the duty to analyze all possible hints for anomalies and to extract as much information as we can from SN1987A neutrinos.

The plan of this paper is the following: in Sect. 2 we discuss the tools we have to separate signal from background events; in Sect. 3 we formulate definite hypotheses on the background and on the signal events, and show that the average energy of SN1987A neutrinos agrees much better with the expectations when we account for the presence of a few background events; in Sect. 4 we comment on the approach we used and the results we obtained, and finally draw our conclusions.

\section{Tools to separate signal from background in Kamiokande-II dataset}

We begin recalling what are the known and the unknown aspects for the analysis of the events observed in KII. Several (though not all) characteristics of the background are known: the events are Poisson distributed with a given rate, they have relatively low energy, and they are more frequent in the border of the volume used in the analysis [5, that touches the planes of the photo-tubes. The characteristics of the signal are, instead, known theoretically: the events are distributed uniformly in the detector, they have relatively high energy, and their rate is not precisely known. Going into details, the characteristics of the background and of the signal that we will use are:

1) The volume of the KII detector used in the analysis of SN1987A neutrinos has

$$
\text { radius of } R=7.2 \mathrm{~m} \text {, total height of } H=13.1 \mathrm{~m} \text {, }
$$


for a volume of 2140 tons. This volume is much larger than the 680 tons fiducial volume defined in [7, where background events are rare. We know that this wide volume is not free from background [5. The energy distribution of the background in our figure 2 is obtained by multiplying the background per unit time given in figure 2a of Lamb and Loredo [22] by the time elapsed from the first to the last events,

$$
T=12.439 \mathrm{sec}
$$

that makes 2.3 background events on average and 0.272 of them above the threshold of $7.5 \mathrm{MeV}$. The assumption on the background has been validated by checking that the distributions of $N_{\text {hit }}$ obtained from figure 4 of [5] and the average number of background events shown in figure 10 of the same KII paper are in good agreement with what we obtain with our distribution. More discussion of the assumed background is in Sect. 2.3 and App. A.

2) The supernova neutrino signal is supposed to be due to $\bar{\nu}_{e} p \rightarrow n e^{+}$('inverse beta decay') reaction. We use the cross section in [15] to perform the integration on the neutrino energy using the full matrix element and kinematical range. The antineutrino flux that we assume is the one described in Sects. I and II of [23], with the parameters of equation 8 there $\left(\bar{\nu}_{\mu}\right.$ and $\bar{\nu}_{\tau}$ average energy $10 \%$ higher than $\bar{\nu}_{e}$ as in [14, energy equipartition and $\alpha=3$ ), including oscillations. The two crucial input parameters are:

$$
\begin{aligned}
\text { energy radiated in } \bar{\nu}_{e} & =4 \times 10^{52} \mathrm{erg} \\
\text { average energy of } \bar{\nu}_{e} & =14 \mathrm{MeV}
\end{aligned}
$$

The first value is just in the middle of theoretical expectations. Instead, the value of the average energy of $\bar{\nu}_{e}$ is on the low side, but compatible with the present theoretical expectations [14. Furthermore, it fits well the IMB observations [2], that consisting of a sample of 8 high energy events are in practice background free. Two simple tests permit us to check that these values are reasonable: a) When the emitted antineutrino energy is compared with the (fermionic) StefanBoltzmann law $L_{\bar{\nu}_{e}}=7 \pi^{3} / 240 R^{2} T^{4}$, it corresponds to an emission from a spherical surface of radius $R=12 \mathrm{~km}$ that lasts 12.6 seconds. b) The energy radiated compares well with LattimerYahil formulae [11, 13]. In fact, assuming the total emitted energy is 6 times larger than the one emitted in antineutrinos (equipartition), the expected value $E_{b}=1.5 \times 10^{53}\left(M_{g} / M_{\odot}\right)^{2}$ erg gives $M_{g}=1.3 M_{\odot}$; then the initial mass of the stellar core is $M_{c}=M_{g}\left(1+0.084 M_{g} / M_{\odot}\right)=1.4 M_{\odot}$. See [23] for further discussion of the selected model. With this model, we expect 11.8 signal events in Kamiokande-II.

With more detailed experimental information, more precise statements could be possible; it would be useful to know the background rate for any single event (taking into account the specific position, number of hit phototubes and photoelectrons, possible correlations, etc); the error on the position of any event; the energy distribution of the two components of the background, namely the one which is located in the border (of higher energy) and the one that is distributed in the volume (of lower energy). With reference to the last point, we quote the KamiokandeII collaboration [5]: the events with $N_{h i t} \geq 23$ "are consistent with higher-energy products of radioactivity at or outside the tank wall" whereas "the events with $N_{\text {hit }} \leq 20$ are largely due to ${ }^{214} \mathrm{Bi}$ decay" (from the ${ }^{222} \mathrm{Rn}$ chain) and thus, presumably, more uniformly distributed. In the present paper we resort only to the published information on the background [5, 6, 7, 21].

In the following of this section, we will obtain some new hints on possible background events without the need of adopting a specific model for neutrino emission. However, when we will pass to the comparison of the expectations and the observed energy distribution, we will adopt the 


\begin{tabular}{|c||ccc|ccc|ccc|ccc|}
\hline & 1 & 2 & 3 & 4 & 5 & 6 & 7 & 8 & 9 & 10 & 11 & 12 \\
\hline$N_{\text {hit }}$ & 58 & 36 & 25 & 26 & 39 & 16 & 83 & 54 & 51 & 21 & 37 & 24 \\
$E[\mathrm{MeV}]$ & 20.0 & 13.5 & 7.5 & 9.2 & 12.8 & 6.3 & 35.4 & 21.0 & 19.8 & 8.6 & 13.0 & 8.9 \\
$\delta E[\mathrm{MeV}]$ & 2.9 & 3.2 & 2.0 & 2.7 & 2.9 & 1.7 & 8.0 & 4.2 & 3.2 & 2.7 & 2.6 & 1.9 \\
\hline$\theta_{\perp}[\mathrm{deg}]$ & 14 & 95 & 40 & 36 & 66 & 137 & 56 & 50 & 39 & 51 & 70 & 106 \\
$D_{\text {out }}[\mathrm{m}]$ & 13 & 0.7 & 16 & 14 & 13 & 2.7 & 13 & 13 & 10 & 0.2 & 10 & 9 \\
$D_{\text {in }}[\mathrm{cm}]$ & 200 & 240 & 13 & 2 & 150 & 1200 & 14 & 5 & 640 & 3 & 450 & 530 \\
$d_{\text {min }}[\mathrm{cm}]$ & 200 & 9 & 10 & 1 & 70 & 200 & 13 & 3 & 500 & 2 & 300 & 300 \\
$f[\%]$ & 37 & 96 & 96 & 99 & 72 & 35 & 94 & 99 & 3 & 99 & 20 & 21 \\
\hline
\end{tabular}

Table 1: Value of certain observables described in the text. First line, progressive event number; first column, the selected observables.

well-specified model for the signal of eq. 3 obtaining in this way other hints on possible background events. At this point, one could think that the conclusions that we reach on background are not reliable, since they depend at least partly on the assumed model for neutrino emission. Thus, in order to avoid confusion, we prefer to repeat that the first purpose of our analysis is to check whether there is a problem with the conventional expectations for the supernova neutrinos, and the second is to ask whether this problem can be solved by making reasonable assumptions on the background. So, the use of a theoretically motivated model to compare with is not only justified, but is in fact a necessary first step. We will stick as much as possible to the very simple model defined above, but will also consider reasonable (theoretically acceptable) modifications from the assumed model, showing that most of the results and the indications we obtain are stable. Of course, if a new paradigm for neutrino emission will eventually emerge, it will be important to update these considerations and repeat the calculations described below.

The tools that we describe in this section are the spatial (Sect. 2.1), the energy distribution of the events (Sect. 2.2) and the energy threshold used by Kamiokande-II collaboration (Sect. 2.3). The compatibility of the indications will be discussed later (Sect. 3).

\subsection{Spatial distribution}

A lot of useful information about each of the 12 events is given in tables I and II of [5]: the number of hit photo-tubes $N_{h i t}$, the reconstructed energy of the events (using additional information) and its uncertainty, the Cartesian coordinates $x_{i}, y_{i}, z_{i}$ (but not the error on the position) and finally the reconstructed direction of the momentum $\cos \alpha_{i}, \cos \beta_{i}, \cos \gamma_{i}$. In this way we can deduce several interesting quantities, e.g.,

$\theta_{\perp}$ the angle between the direction of the momentum and the normal to the closest surface of the volume (events with $\theta_{\perp} \sim 0$ are presumably seen better);

$D_{\text {out }}$ the distance between the coordinate of the event and a formal 'exit point' from the volume, following the direction of the momentum $\left(D_{\text {out }}\right.$ should be large in comparison to the distance between photo-tubes, about 1 meter, for an event to be reliably reconstructed);

$D_{\text {in }}$ the distance between the coordinate of the event and a formal 'entry point' in the volume, following again the momentum but in the opposite direction; 


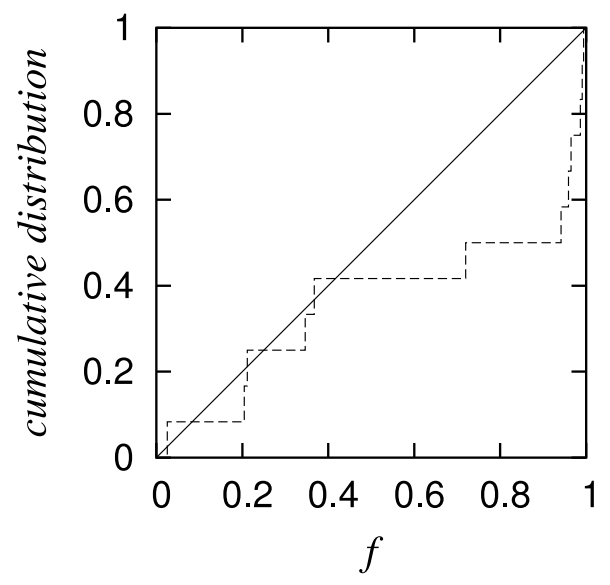

Figure 1: The volumetric distribution of the 12 KII candidate supernova neutrino events (dashed line) compared with a uniform distribution (continuous line).

$d_{\text {min }}$ the minimal distance from the border of the volume used in the analysis (that can warn us against possible background events);

$f$ the volumetric coordinate, namely the fraction of the volume contained in a concentric subdetector of the same shape and with the same geometrical center of KII, that is formally defined as follows:

$$
f_{i}=\max \left[\frac{\sqrt{x_{i}^{2}+y_{i}^{2}}}{R}, \frac{\operatorname{abs}\left(h_{i}\right)}{H / 2}\right]^{3}
$$

where we set $h_{i}=z_{i}-60 \mathrm{~cm}$, taking into account the offset of the origin of the coordinates.

The last quantity is of particular interest, since when we increase the volume of the sub-detector, the number of supernova neutrino events should increase by the same factor (i.e., $f$ can be considered as the cumulative distribution coming from a uniform probability distribution function).

In App. B we show that, assuming uniformity (namely ignoring systematics effects due to the detector response) we are underestimating the impact of a possible contamination from relatively high energy background events, that are known to be preferentially located in the border of the detector [5]. However, before proceeding we wish to stress the importance of a detailed quantitative study of the detector response to a sample of uniformly distributed supernova neutrino events (including fluctuations, realistic light propagation, role of the energy spectrum, imprecise measurement of the position, etc.) and we also warn the reader against the possibility of significant systematics effects, of which we are not aware.

As can be seen in table 1, there is no particular feature of the observable $\theta_{\perp}$. Similarly for the distance $D_{\text {out }}$, which is usually large, except for the events 10 and 2. Anomalies emerge instead in the distributions of $D_{i n}$, of the minimal distance from the wall $d_{\min }$ and of the volumetric coordinate $f$ : all these show that there are events quite close to the surface of the volume. In particular, $d_{\text {min }}$ is smaller than 10 centimeters for the events $2,3,4,8$ and 10 and $f$ shows that the same events are contained in the outermost $4 \%$ part of the 2140 ton volume, see 
figure 1. Performing a Smirnov-Cramèr-Von Mises (SCVM) [27, 28, test for the hypothesis that all 12 events come from a uniform distribution we get a goodness of fit of $4.6 \%$. When we perform a test suited to reveal features in the border, this hint for a deviation from a uniform distribution becomes stronger. In fact, the goodness of fit is $0.3 \%$ with the traditional version of the Anderson-Darling (AD) [27, 29] test, whereas the one sided version of this test gives $0.04 \%$ : see App. Cf for details. Attributing the event number 6 to background the goodness of fit becomes even lower, for the simple reason that this event is not located in the border. We can regard these results as a suggestion that there is contamination from background events located close (or at) the border of the volume that was used for the analysis. We interpret this as a $3 \sigma$ hint, with reference to the numerical value of the traditional AD test.

It is important to repeat that the assumption of a uniform distribution of neutrinos is valid whatever was the true (energy and/or time) distribution of SN1987A neutrinos. To the best of our knowledge, the analysis of the spatial distribution presented here and elaborated further in App. C] and Sect. 3 is original. In [1] we read that "the distribution of the events presented here is consistent with a uniform distribution", but unfortunately, the procedure adopted, the statistical test performed and the significance level are not indicated, so it is not possible to compare the results.

\subsection{Energy distribution}

In this section, we compare the observed and expected energy distributions. In the first part (section 2.2.1) we assess on quantitative basis the 'problem' of the energy distribution, in the second part (section 2.2.2) we discuss the probabilities that the individual events to be due to background, that we then use to make statements on the expected number of events and on significance of the observations.

\subsubsection{Comparison of the expected and the measured energy distributions}

The expected event distribution is the continuous, bimodal curve in figure 2, The two distinct components are simply the (measured, low-energy) background and the (assumed, high-energy) signal due to supernova neutrinos, discussed in the beginning of Sect. 2. Clearly, the agreement of the theoretical distribution (the continuous curve in figure 2) with the observations (the dashed curve in figure 2) could be improved if the theoretical parameters were very different from what we expect, as assumed in [25] and [26]. In fact, one could be tempted to argue from figure 2 that it is necessary to assume a significantly lower value of the average energy of neutrinos than assumed in equation 3. But, is it really correct to conclude that the apparent disagreement is not due to a fluctuation? We performed a standard SCVM test for the null hypothesis described in Sect. 2 (or in figure 2) finding that

the assumed energy distribution (measured background + expected signal) should not be rejected at the $11 \%$ significance level.

Thus, we believe that we are justified in assuming that the theoretical distribution (that was motivated previously) is not in contradiction with KII observations.

Before proceeding, however, it is useful to explain better in which sense one could claim the existence of a 'problem' with low energy SN1987A neutrinos. Suppose we ask whether the KII data could come from the assumed signal setting the background to zero; using again a SCVM 


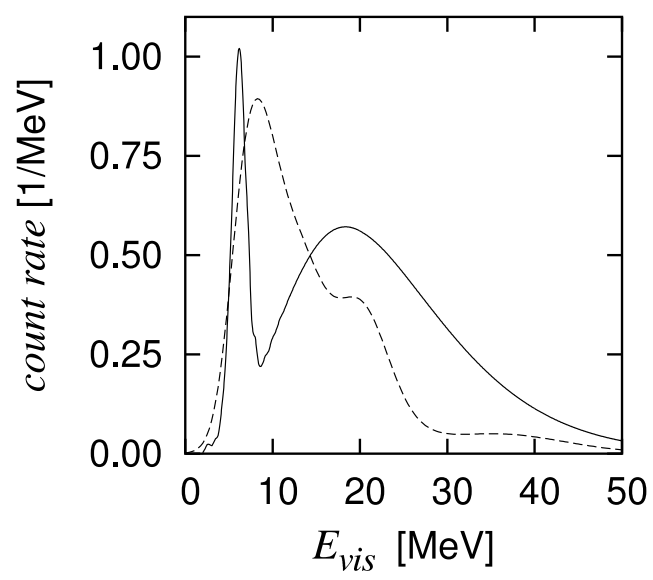

Figure 2: The continuous, bimodal curve is the count rate expected in KII from the observed background (low energy peak) and the supernova neutrino signal, see text for a description. For visual comparison, we show also the spectrum obtained summing the Gaussian energy distributions of the 12 observed events, with $E_{i}$ and $\delta E_{i}$ as given in table 1 (dashed curve).

test, we find that this hypothesis can be accepted but only at $0.4 \%$ significance level $(2.9 \sigma)$. In other words, we find that the assumed signal is in disagreement with the data if we posit that the background is absent. However, in view of the preceding discussion, we believe that this position is hardly tenable and, as we demonstrated here, it has an important impact for the interpretation of the data. So, we assume the concomitant presence of signal and background. The next step is to explore what are the quantitative implications of this position, in particular, for what concerns the background 3

\subsubsection{Analysis of the individual events}

In Čerenkov type detector it is not possible to state whether a single event is due to signal or to background. However, knowing the energy distribution of the signal and the one of the background, it is possible to assign to each event a probability $P_{i}$ that it resulted from background (and a probability $1-P_{i}$ to result from signal).

This can be done proceeding as follows. The probability per unit second $B_{i}$ (background rate) that an event with energy $E_{i}$ results from background is known experimentally. The data that we use and the numerical values of the $B_{i}$ are described in App. $\mathrm{A}$. In order to obtain the signal rate, instead, we describe each event with a Gaussian distribution centered at the energy

\footnotetext{
${ }^{3}$ One may ask why we do not proceed similarly for the spatial distribution. In Sect. 2.1 we test only whether a subset of data is uniformly distributed (i.e., whether the subset can be attributed to supernova neutrino events), here we give the energy distribution of the signal plus the background and compare with the observations. The reason of this asymmetric procedure is simply that we do not have the a priori information on the spatial distribution of the background, as recalled in the beginning of Sect. 2 we only know that the events with $N_{\text {hit }} \geq 23$ "are consistent with higher-energy products of radioactivity at or outside the tank wall" and we keep in mind the message of figure 5 of [5].
} 
of the event $E_{i}$ and with a width $\delta E_{i}$ as measured:

$$
\rho_{i}(E) \propto \exp \left[-\frac{\left(E-E_{i}\right)^{2}}{2 \delta E_{i}^{2}}\right]
$$

Next, we calculate the convolution integral $\mathcal{I}_{i}$ of this Gaussian with the expected distribution of the signal. We obtain the corresponding signal rate $S_{i}=\mathcal{I}_{i} / T$ simply dividing by the time $T$ from the first to the last event given in eq. 2, In this way we can compare the expectations for the background and for the signal, and we can calculate the adimensional quantity

$$
P_{i}=\frac{B_{i}}{B_{i}+S_{i}}
$$

that can be thought of as the probability that an event is due to background. The approximate values of $P_{i}$ are given below (0 means 'small' with the given precision):

\begin{tabular}{c||ccc|ccc|ccc|ccc} 
event & 1 & 2 & 3 & 4 & 5 & 6 & 7 & 8 & 9 & 10 & 11 & 12 \\
$P_{i}$ & $0 \%$ & $1 \%$ & $74 \%$ & $29 \%$ & $1 \%$ & $93 \%$ & $0 \%$ & $0 \%$ & $0 \%$ & $51 \%$ & $1 \%$ & $43 \%$
\end{tabular}

This table indicates that the events $3,4,6,10,12$ have a non-negligible chance to be due to background, due to their relatively low energy.

These results do not change much when we modify the energy distribution in a manner that does not contradict the theoretical expectations, e.g., when we use $\alpha=2$ rather than 3 , or when we diminish by a $1-2 \mathrm{MeV}$ the average energy of supernova neutrinos. E.g., if the average neutrino energy were $12 \mathrm{MeV}$ (resp., if $\alpha=2$ ), the convolution integral $\mathcal{I}_{10}$ would increase by $20 \%$ (resp., decrease by $10 \%$ ), and thus $B_{10}$ would decreases, but only by $10 \%$ (resp., increase by $5 \%$ ). It is pretty much evident that the probability that a low energy event is due to background remains quite large if the signal resembles the one shown in figure 2 ,

It should be noted that the number of background events above the threshold of $7.5 \mathrm{MeV}$, that we evaluate a posteriori using the selected model of supernova neutrino emission, is seven times larger than the value expected a priori. In other words, even if postulating the presence of a pair of background events is the best comprimise we can reach with the model, this is not necessarily a good compromise; this could be alternatively interpreted as an indication that the conventional model for neutrino emission that we adopted misses important features of the data. More discussion later.

Armed with these results, we find that the probability that all events are signal is just $0.3 \%$, whereas (as obvious) it is basically impossible to have a fluctuation of 12 events that has these characteristics. We calculate on this basis a significance of about $10 \sigma$ that at least one of these events are due to supernova neutrinos, that is higher than the $6.5 \sigma$ significance that can be estimated from figure 10(b) of [5] (using only the information on the background). Though this result is based on the tails of the energy distributions, and should not be fully trusted quantitatively, it carries a reasonable and reassuring message: if we include the information on the expected signal, the evidence for a detection of neutrinos from SN1987A strengthens.

The most interesting and probable cases have several background events:

\begin{tabular}{c||ccccc} 
\# of bkgr. events $n$ & 1 & 2 & 3 & 4 & 5 \\
probability $\mathcal{P}_{n}$ & $6 \%$ & $26 \%$ & $39 \%$ & $23 \%$ & $5 \%$
\end{tabular}


This is easily evaluated by constructing the polynomial $q[x]=\Pi_{i}\left(1-P_{i}+x P_{i}\right)$ since, if $P_{i}$ is the background probability, $1-P_{i}$ can be thought of as the signal probability. The coefficient $\mathcal{P}_{n}$ of $x^{n}$ counts the probability to have exactly $n$ background events; thus we should simply expand the polynomial $q[x]=\sum_{n} \mathcal{P}_{n} x^{n}$.

Similar results are presented in table VI of [21]. But in that work the main issue is the study of the time distribution of neutrinos, whereas the average energy of supernova neutrinos is considered a free parameter whose value is decided from a global fit. Here, in order to discuss whether KII observations necessarily indicate an excess of low energy neutrinos, we followed a different approach, and adopted a (fixed, pre-selected) value of the $\bar{\nu}_{e}$ average energy that is compatible with the calculated ones and with IMB observations. Other comparisons with the reference results obtained by Lamb and Loredo are presented in the following.

Let us repeat that the expectations on the background here discussed are obtained a posteriori adopting a pre-selected model of supernova emission. The a priori expectation on the background are discussed in the next Section, and a detailed comparison of the outcomes is offered in table 2 and discussion given there.

In summary, we verified: (1) that the observations do not contradict seriously the theoretical expectations on the energy distribution of supernova neutrinos; (2) that this distribution, along with the measured energy distribution of the background, suggests the hypothesis that 2 to 4 low energy events in KII dataset are due to background.

\subsection{Energy threshold}

The criterion adopted by Kamiokande-II collaboration 1 to separate the signal from the background is the energy threshold of $7.5 \mathrm{MeV}$ (corresponding to about $N_{\text {hit }}=20$ ) used in solar neutrino analyses. In fact, in the abstract of [1] we read: "the signal consisted of 11 electron events of energy 7.5 to $36 \mathrm{MeV}$ "; in the text: "event number 6 has $N_{\text {hit }}<20$ and has been excluded from the signal analysis". In our opinion, this criterion is perfectly fine if the aim of the analysis is to claim that there is an excess that cannot be explained as a fluctuation of the background; it is less fine if the aim is to investigate the properties of the signal.

We propose a number of critical remarks on the exclusive use of this criterion:

1) A priori, there is no warranty that a quantitative criterion that works well for solar neutrinos works also for supernova neutrino signal. In fact, solar neutrino events are directional, whereas $\bar{\nu}_{e} p \rightarrow n e^{+}$events are not, the interaction rates of solar and supernova neutrinos are not the same, and the energy distributions are also different. However, when this criterion is adopted and when a time window is selected one can check the expected number of background events above threshold: from fig. 10 of [5], we deduce that in the time window spanned by the 12 events, eq. 2, this makes 0.272 events. Though this is not very large, it is not completely safe too.

2) In general, the choice of a criterion to distinguish signal and background is not independent from the signal we want to reveal. For instance, the model for supernova neutrinos considered here suggests that the criterion of setting the threshold at $7.5 \mathrm{MeV}$ is insufficient to ensure that, above, we have only supernova neutrinos: in fact, we find that the background is smaller than $5 \%$ only above $10 \mathrm{MeV}$, corresponding to about $N_{\text {hit }}=26$ (see figure 2).

3) Finally, it is possible to argue against the procedure of excluding only the event number 6 from the supernova dataset: In fact, removing the events 6 , the confidence level that we have a uniform volumetric distribution halves when we use the AD or the SCVM test (see App. C and later on). In other words, the likelihood diminishes significantly unless other signal events are 
also assigned to background. It should be clear that this last argument is completely independent on the model of supernova neutrino emission.

While we have no reservation to accept the hypothesis that the event 6 is due to background (see App. A), the arguments above suggest that it could be not the only background event in the time window of eq. 2. As we discussed in the previous section, we believe that the best approach to extract and study the signal is to quantify the probability that an event is due to background; however, it is useful and instructive to consider also the simpler criterion of setting the energy threshold at $7.5 \mathrm{MeV}$ (the comparison of these criteria will be done in Sect. 3.1.1). More specifically, we will use two versions of this criterion:

V1 In the first version, we simply discard the event number 6 , and consider the events that fall under the threshold within $1 \sigma$ (namely, the events $3,4,10,12$ ) as suspect. Thus, we will attempt to attribute some of them to background, in order to see if the volume distribution improves or not.

V2 In the second version, we will do the same, except for assigning an additional penalty factor each time we attribute one of these four events to background. The penalty factor is simply the Poisson probability for $n$ background events, where the average value is set to $b=0.272$ (for instance, the penalty factor to have 2 such events is $2.8 \%$ ). In this way, we somehow describe what we know on energy distribution of the background, though we are ignoring any a priori information on the energy distribution of the signal.

These two versions of the criterion are used in table 3 of the next Section; see also the second line of table 2 ,

\section{Average energy of SN1987A neutrinos accounting for background}

As we repeatedly argued, in order to address the question on supernova neutrinos raised in the title of the paper we deem it necessary to discuss the role of background events in KII dataset. Here we collect the available information for such a discussion and draw our conclusions on whether there is a problem with low energy SN1987A neutrinos. As discussed in Sect. 2, we will assume that all the events except those that are assigned to background are due to $\bar{\nu}_{e} p \rightarrow n e^{+}$ events (an extension of the 'inverse beta decay hypothesis'). Thus, the identification of the events due to SN1987A neutrinos becomes equivalent to the identification of the background events 4

The first task is to identify the main candidate background events, and for this aim we use the tools of Sect. 2. We begin listing again (a) the events that are closer to the border, namely: $2,3,4,8,10$ and (b) the events with relatively low energy: $3,4,6,10,12$ with $N_{\text {hit }}$ equal to 25 , 26, 16, 21, 24. Thus, we select the events $3,4,6,10,12$ for further discussion:

\footnotetext{
${ }^{4}$ Other approaches are in principle possible. For what concerns misidentification of signal against background, one could decide to accept only the events in the fiducial volume; if we use the definition of [7] we are led to keep only the five events 1, 6, 9, 11, 12. A similar possibility would be to reject all events under a 'fiducial threshold' that, according to our expectations, should be around $10 \mathrm{MeV}$ (see Sect. 2.3); again in this way we would remove several events including one of the previous subset. Both procedures should provide us with relatively safe results, but would amount to largely diminish the information on the characteristics of the supernova burst that we can extract from KII. In view of the small dataset, we believe that the most useful approach is to make an assessment on the background.
} 


\begin{tabular}{|l||c|cc|cc|c|c|}
\hline Events removed & none & 6 & 10 & 6,10 & 3,10 & $3,6,10$ & $3,4,6,10$ \\
\hline (a) bkgr. above $E_{t h}$ & $76 \%$ & $76 \%$ & $21 \%$ & $21 \%$ & $3 \%$ & $3 \%$ & $0.3 \%$ \\
(b) Vol.distr., SCVM & $4.6 \%$ & $2.4 \%$ & $12 \%$ & $7.2 \%$ & $26 \%$ & $18 \%$ & $42 \%$ \\
(b) Vol.distr, AD-2side & $0.3 \%$ & $0.1 \%$ & $2.2 \%$ & $1.0 \%$ & $6.9 \%$ & $3.8 \%$ & $21 \%$ \\
(b) Vol.distr, AD-1side & $.04 \%$ & $.02 \%$ & $0.4 \%$ & $0.2 \%$ & $1.5 \%$ & $0.8 \%$ & $7.8 \%$ \\
(c) Energy distribution & $0.3 \%$ & $4.6 \%$ & $0.3 \%$ & $4.7 \%$ & $1.0 \%$ & $14 \%$ & $5.6 \%$ \\
\hline (d) Aver. energy [MeV] & $15 \pm 2.3$ & $15 \pm 2.4$ & $15 \pm 2.4$ & $16 \pm 2.5$ & $16 \pm 2.5$ & $17 \pm 2.6$ & $18 \pm 2.7$ \\
\hline
\end{tabular}

Table 2: Impact of various assumptions on the background events; first row, the events assigned to background sample; second line, the Poisson probability of $n$ events above threshold of $7.5 \mathrm{MeV}$; third line, the SCVM significance level of a deviation from a uniform volumetric distribution; fourth line, the same using the two sided (traditional) AD statistic; fifth line, the same using the one sided (modified) AD statistic; sixth line, the probability of the given energy configuration, evaluated with the considerations of section 2.2; last line, average visible energy of the supernova signal events.

6 This is the lowest energy event and it has a high probability to be due to the 'diffuse' component of the background (e.g., internal radioactivity, radon, cosmic ray induced or neutrons). Being under the threshold of software analysis, $N_{\text {hit }}=20$, it is removed in most investigations of supernova neutrinos (see Sect. 2.3 and App. A).

10 Besides being a low energy event, this event is known to travel a few tens of centimeters in the 2140 ton detector volume and to be very close to the lateral surface and to the upper plane of the detector-it is in the edge of the volume. We are not able to assign a strong quantitative significance to this information, but we believe that it puts a second red herring on this event.

3, 4 These two events are quite similar; they both have low energy and are both very close to the border of the 2140 ton volume used in the analysis. Thus, rejecting one or the other from the supernova neutrino sample produces a similar effect.

12 This is just the last event, with an energy similar to the event number 10, and thus (depending on the true time distribution) could fall in a region where few signal events are expected. It is located far from the border, though.

\subsection{A first global approach}

Now we attempt to attribute some of the events mentioned above to background, testing whether the spatial and the energy distributions of supernova neutrinos improve or not. The results are shown in table 2, where we give: (a) the Poisson probability to have a certain number of background events above the threshold (see Sect. 2.3); (b) the significance levels assuming a uniform volumetric distribution, evaluated respectively using the SCVM and the two AD statistics described in App. C (see also Sect. 2.11); (c) the probability of a given distribution of signal and background, that in the case where all events are declared to be signal is $\Pi_{i}\left(1-P_{i}\right)=$ $0.32 \%$, but e.g., increases by the factor $P_{6} /\left(1-P_{6}\right)=14$ removing the event number 6 (see Sect. 2.2); (d) the average energy of the signal sample, that is the aim of our discussion. 


\subsubsection{Model dependent and independent inferences}

The only really model independent test that we are aware of is the test for a uniform distribution of supernova neutrinos. The most powerful test between the Smirnov-Cramèr-Von Mises and the Anderson-Darling test is the second one. In fact, this is built to reveal deviations from the expectations close to the boundaries, that could mean the presence of background events. We will use this test in the following, alone and in combination with other ones. We will also use the SCVM and compare the outcome of various procedures of analysis, reaching in all cases similar conclusions. These conclusions will be taken as a guide to formulate a hypothesis on the background and on the signal of KII dataset.

Analysis A First, we present the results of the one sided (modified) Anderson-Darling test described in App. C. With the conventional $5 \%$ significance level, the only case among those of table 2 that should not be rejected is the one where all four candidate background events are attributed to background (see fifth row of table 22). Another interesting conclusion that we obtain from the one sided AD test is that at least 3 events close to the border should be attributed to background. The conclusions till here are independent on the energy distribution. When we use the a priori expectations on the energy distribution of the background we conclude that the lowest energy events, among those selected by the AD test, have a higher chance to be due to background. This is the closest we can go to a model independent argument.

Analyses B1,B2,B3 Next, we quantify the improvements (likelihood ratios) in the description of the supernova neutrino signal under the various hypotheses on the background described in Sect. 3. We will compare four different procedures. In all of them, we perform a traditional (two sided) AD test to check the uniformity of the assumed supernova events in the volume. Additionally:

B1) we follow Kamiokande-II and assign a priori the event number 6 to background (see Sect. 2.3. first version of the threshold criterion);

B2) additionally to B2, we impose a Poisson penalty for background events above the threshold of $7.5 \mathrm{MeV}$ (see Sect. 2.3, second version of the threshold criterion);

B3) we test the likelihood of the given energy distribution as in Sect. 2.2.

With any of the four procedures we evaluate the factors of improvements of the given hypothesis, choosing as a comparison the case when all 12 events (or 11 events in the second case) are considered to be due to supernova neutrinos. E.g., the procedure B3 suggests that the hypothesis that only the event 6 is due to background is more probable than the hypothesis that all events are signal, by a factor of improvement equal to

$$
\frac{P_{\text {vol. }}(\text { all events but } 6) \times P_{\text {en. }}(\text { all events but } 6)}{P_{\text {vol. }}(\text { all events }) \times P_{\text {en. }}(\text { all events })}=5.7
$$

(approximate values of $P$ are given in table 21). The results of these calculations are given in table 3 ,

The procedure B1 makes very little use of the background and of the signal; the procedure B2 uses the a priori knowledge on the background, but neglects any information on the signal; the procedure B3 uses all we know, though its reliability depends also on the model for supernova neutrinos we assume. It is difficult to make firm conclusions from this table, but we note that even in the more pessimistic procedure (B2) the presence of several background events cannot 


\begin{tabular}{|c||c|c|c|c|c|c||c|}
\hline AD-2side + & 6 & 10 & 6,10 & 3,10 & $3,6,10$ & $3,4,6,10$ & remarks \\
\hline Sect. 2.3. V1 & $\equiv 1$ & - & 8.2 & - & 30 & 160 & model indep. \\
Sect. 2.3 V2 & $\equiv 1$ & - & 2.2 & - & 1.1 & 0.5 & model indep. \\
Energy distrib. & 5.7 & 7.0 & 48 & 64 & 500 & 1100 & model dependent \\
\hline
\end{tabular}

Table 3: Factors of improvement in the description of the Kamiokande-II supernova neutrino events for the selected hypotheses on the background. The subset of events that are assigned to background is in first line. In addition to the Anderson-Darling test for a uniform volumetric distribution, we use the information listed in the first column. When we impose the Kamiokande-II threshold (second and third rows) the event 6 is discarded a priori from the supernova dataset. Last column, comments on the selected procedure.

be firmly excluded; this is because an a priori unlikely fluctuation of the number of background events above threshold is compensated by the fact that some of these events lie close to the border of the detector. On the other hand, we see that with the procedure of analysis B3, the only one that makes a full use of the knowledge on the background and on the signal, the factors of improvements are about a thousand times when all 4 candidate background events are assumed to be background, and half of that value when we assign events 3, 6, 10 (or 4, 6, 10) to background. In short, table 3 suggest that a compromise between the extreme assumptionsnamely, of considering completely unknown or reasonably known the energy distribution of the signal-would admit the presence of several background events. It is important to note that the results of the more aggressive procedure of analysis (B3) do not change much when we modify the energy distribution in a manner that does not contradict the theoretical expectations, see Sect. 2.2. In other words, one could be tempted to argue that the model dependence of our stronger analysis is not large, if the astrophysical parameters lie in the range suggested by the theory and by the IMB observations: see again Sect. 2] and [23].

Analyses C1,C2 Finally, one may ask what happens when we apply the SCVM test for the spatial distribution of the events, instead than the AD test that we used so far, or when we change the procedure of analysis. We show that the conclusion is very similar to the previous ones, though it is true that the weight of the energy distribution becomes comparably more important with the SCVM test.

Let us begin with a description of the procedure C1. According to our hypothesis, any of the 12 events can be due either to background or to inverse beta decay signal. Thus we consider the $2^{12}=4096$ individual possibilities, and for any of them we test the likelihood of the given spatial distribution, using this time the SCVM test and the likelihood of the given energy configuration as we did in Sect. 2.2 (we checked that with a SCVM test we obtain similar outcomes). Next, we consider the product of the two likelihoods and normalize the sum of the likelihoods of the 4096 mutually exclusive cases to unity. We find that the occurrence of several background events is likely:

$$
\begin{array}{c||ccccccc}
\text { \# of bkgr. events } n & 0 & 1 & 2 & 3 & 4 & 5 & 6 \\
\text { probability } & 0.1 \% & 2.2 \% & 14.6 \% & 37.3 \% & 33.8 \% & 11.4 \% & 0.5 \%
\end{array}
$$

The individual cases with probability above $5 \%$ are the following ones: 

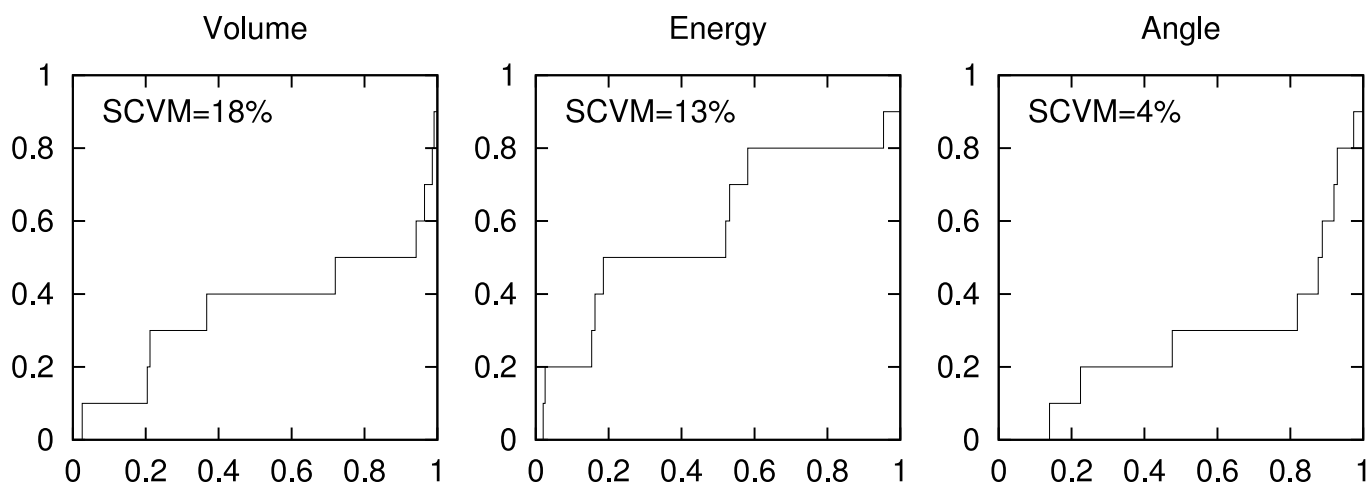

Figure 3: Cumulative distributions for the case when the events number 3, 4, 6 are assigned to background, and significance of the SCVM test. In the abscissa the value $F_{j}$ of the cumulative distribution (see App. (C).

\begin{tabular}{c||c|c|c|c|c|c|} 
background events & $3,6,10$ & $3,4,6,10$ & $3,4,6,10,12$ & $3,6,10,12$ & $3,4,6$ & 3,6 \\
probability $\mathcal{P}_{n}$ & $19.1 \%$ & $18.3 \%$ & $9.9 \%$ & $8.1 \%$ & $7.7 \%$ & $6.8 \%$
\end{tabular}

We note that the event number 12 is occasionally assigned to background because of its low energy. What about the event number 6 ? In all those cases that have probability larger than 3 $\%$, this event is due to background. We also remark that the 2048 cases when this event is due to background have a total probability of $90 \%$.

In the procedure of analysis C2 we test also the SCVM likelihood of the angular distribution following [23]. The events that are most commonly assigned to background are the usual ones, namely those figured out in the beginning of Sect. 3. The individual cases with probability above $5 \%$ (the most probable individual cases) are those when the background is given by the events number 3,4,6 (15.4\%), 3,6 (14.0\%), 3,6,10 (11.9\%); 3,4,6,10 (9.1\%), where in brackets we give again their a posteriori probabilities, that by definition are normalized to give 1 when we sum over the 4096 possible cases. The cumulative distributions in the volume, in the energy and in angle for the most probable single case (the first one listed just above) are shown in figure 3 , along with the likelihood of the three experimental distributions. Remarks on the persistent discrepancy of the angular distribution will be offered in Sect. 4. The overall probabilities to have 2, 3 or 4 background events becomes $28 \%, 41 \%$ and $20 \%$ and the probability that all events are due to signal is $0.5 \%$.

\subsubsection{A tentative explanation of low energy events}

In view of the arguments above discussed, we formulate our hypothesis on the signal and on the background:

9 (or less) among the 12 events observed in Kamiokande-II were due to SN1987A neutrinos, whereas the remaining 3 (or more) low energy events were due to background.

The motivations and the merits of this position are discussed in Sect. 3.1.1.

Quite importantly, this hypothesis allows us to argue that there is no excess of low energy supernova neutrinos. Consider as a quantitative indicator the average visible energy of supernova neutrino events, that being weighted with the detection efficiency and the cross section is 
significantly higher of the average antineutrino energy-see, e.g., 23]. With the signal described in the beginning of Sect 2, the average visible energy of supernova neutrino events is expected to be

$$
E_{v i s}=20.6 \mathrm{MeV} \text {. }
$$

This can be compared with the value given from the data, using the formula

$$
\langle E\rangle \pm \sqrt{\frac{\left\langle E^{2}\right\rangle-\langle E\rangle^{2}}{n}}, \quad\left\langle E^{a}\right\rangle \equiv \sum_{i=1}^{n} \frac{E_{i}^{a}}{n}
$$

where $n$ is the number of events due to supernova neutrinos. If we assume that all events are due to signal $(n=12)$, we find that an average energy that is $2.6 \sigma$ lower than the expectations. This discrepancy is very similar to the $2.9 \sigma$ 'problem' of low energy neutrinos that was formulated in the beginning of Sect. 2.2 one can say that the selected observables catches the crucial feature of the 'problem'. Next we deduce the value of the average visible energy of supernova neutrino events from the data, in the hypothesis on the signal formulated just above. In the case when we assign the events $6,10,3$ (resp., $6,10,3,4)$ to the background the average visible energy of supernova neutrino events is 1.4 (resp., 0.9) standard deviations below the expected value (approximate values are in the last line of table 2). In other words, they are in reasonable agreement. Thus, if 3 or 4 low energy events were caused by background the question raised in the title of the paper should receive a negative answer.

\subsection{A second global approach}

The previous interpretation leaves something to be desired. In fact, we expect only 0.272 background events above the threshold of $7.5 \mathrm{MeV}$ on average in the time between the first and the last event, so there was only a $3 \%$ probability a priori that 2 or more such background events occurred (see third row of table 2). In other words, even if the invoked background fluctuation cannot be just dismissed, it is significantly larger than a priori expected. Furthermore, the event number 10 is in a region of the detector much noisier than on average (compare table 1, Sect. 3 and fig. 5 of [5]) but the events 3 and 4 seem to be located in a relatively safer region. In other words, the hypothesis discussed previously is the best one within the considered theoretical context, but it is not particularly good: this can be seen quite clearly from figure 2, that shows that the low energy events we are discussing are just in between the signal and the background distributions. So, we are motivated to consider whether we can evade the conclusion we reached and/or how we can reach a more satisfactory conclusion. We will show that in order to address at least partially these needs it is sufficient to consider a more accurate model for the supernova neutrinos, in particular, for what regards the time evolution of the neutrino luminosity. This possibility is quite appealing, since it is largely within the conventional expectations.

\subsubsection{A more refined hypothesis on neutrino luminosity}

A phase of intense neutrino luminosity is expected during the first hundred of milliseconds from the collapse. This phase has a marked non-thermal character [8] and it is thought of as to play a key role for the explosion [9, 10, [18. In the following we term this hypothetical phase as "accretion", and the subsequent thermal phase as "cooling". The latter phase is the one considered till now. For the cooling phase, we will continue to assume equipartition, the same 


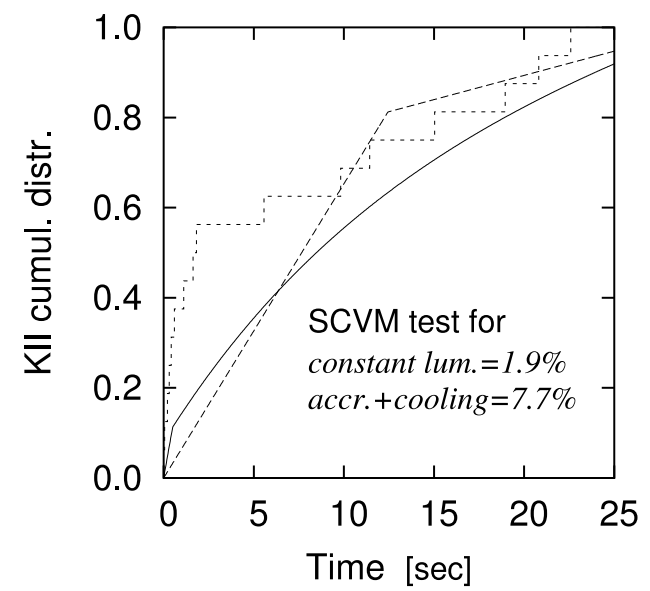

Figure 4: Cumulative time distribution for the 16 events seen by KII in a time window of 30 seconds, compared with the expected distributions for an emission with constant luminosity (long dashed) and with a 'standard' accretion phase (continuous line).

amount of emitted energy, the same average energy as in eq. 3. and an emission time of the order of the time from the first to the last event ( $T$ in eq. 2). Accretion instead lasts just a fraction of second and is much more luminous. We further assume that during accretion equipartition is violated by an excess of electronic neutrinos and antineutrinos: to be definite, we suppose that, on average, there are $3 \nu_{e}$ and $2 \bar{\nu}_{e}$ each $\nu_{\mu}, \nu_{\tau}, \bar{\nu}_{\mu}$, or $\bar{\nu}_{\tau}$. We describe the $\bar{\nu}_{e}$ luminosity in the simplest manner we can conceive:

$$
\mathcal{L}(t)=\left\{\begin{array}{lll}
\frac{\mathcal{E}_{a}}{\tau_{a}} & \text { for } t<\tau_{a} & \text { ("accretion" or non-thermal phase }) \\
\frac{\mathcal{E}_{c}}{\tau_{c}} \times e^{\frac{\tau_{a}-t}{\tau_{c}}} & \text { for } t>\tau_{a} & \text { ("cooling" or thermal phase })
\end{array}\right.
$$

where we select reasonable values for the parameters, namely $\mathcal{E}_{a}=8 \times 10^{51} \mathrm{erg}$ and $\tau_{a}=0.5 \mathrm{~s}$ for accretion, and $\mathcal{E}_{c}=4 \times 10^{52} \mathrm{erg}$ and $\tau_{c}=T$ for cooling. With these numerical values, we can derive several other interesting quantities: 1) the total emitted energy $\mathcal{E}_{b}=4.5 \mathcal{E}_{a}+6 \mathcal{E}_{c}=2.76 \times 10^{53}$ erg; 2) the fraction emitted during accretion $4.5 \mathcal{E}_{a} / \mathcal{E}_{b}=13 \%$; 3 ) the electronic lepton number emitted during accretion, that corresponds to about half solar mass of iron if the average energy of $\nu_{e}$ is of $\left.12 \mathrm{MeV} ; 4\right)$ the energy of 20 times $10^{51} \mathrm{erg}\left(=20\right.$ foe $=20$ bethe) emitted in $\nu_{e}$ and $\bar{\nu}_{e}$, that could be sufficient for the explosion. None of these numbers should be taken too literally, but they are comfortably within the present theoretical expectations.

Let us come now to the interpretation of the events. Since the average energy is supposed to stay unchanged and the total emitted energy is almost the same, the total number of supernova events and their energy distribution do not change much: only their time distribution changes. Before explaining why this position is relevant to the interpretation of the low energy KII events, we begin noticing that the expected rate of event accumulation,

$$
N(<t)=0.187 t+ \begin{cases}2.0 \frac{t}{\tau_{a}} & \text { for } t<\tau_{a} \\ 2.0+11.8\left(1-\exp \left[\frac{\tau_{a}-t}{\tau_{c}}\right]\right) & \text { for } t>\tau_{a}\end{cases}
$$


compares reasonably well with the KII data, since the (SCVM) GOF is $7.7 \%$. In the previous equation we show the contributions of the background-first term-and of the signal separately; the number of events during cooling is the same as before, 11.8. For comparison, the hypothesis that the signal accumulation rate is $11.8 / T$ for $t<T$-that is, supernova neutrinos are emitted with a constant luminosity in an interval of $T$ seconds-has a GOF of $1.9 \%$ only. See figure 4 , where we show the sequence of events observed by Kamiokande-II in a time window of 30 seconds. The last events are well explained by the expected background curve; instead, the remarkable feature of data close to $t=0$ is only partially explained by our assumption that a standard accretion phase of neutrino emission also occurred. Indeed, the expected number of events during accretion is only 2.0 (a bit less than the value $2.4=11.8 \times \mathcal{E}_{a} / \mathcal{E}_{c}$ due to oscillations). If it was possible to double the number of events during accretion, the GOF would increase to $30 \%$. The other parameters of emission play a less crucial role for the subsequent discussion 5 Similarly, if we would arbitrarily omit the event number 3 and the event number 6 from the dataset, the GOF would increase to $25 \%$. For other recent analyses of time distribution that also find an evidence of an initial luminous phase see [20, 21, 24]. Now, we pass to discuss the impact of the updated hypothesis on the interpretation of the events.

\subsubsection{Accretion and the meaning of the early events}

The theoretical context defined in section 3.2.1 suggests strongly the possibility that some of the early events seen in KII occurred during a phase much more luminous than average: $\mathcal{E}_{a} / \tau_{a} \sim$ $5 \times \mathcal{E}_{c} / \tau_{c}$. Identifying, arbitrarily but not unreasonably, the arrival of the first neutrino to the Earth with the time of first detected event, this possibility concerns the events number 1,2,3,4. Thus, the analysis of section 2.2 should be updated, taking into account that the signal rate in the denominator depends explicitly from the time $t_{i}$ at which the individual event occurred. More precisely, the factor $S_{i}$ in equation [6 should be multiplied by $\xi\left(t_{i}\right)$ that depends on the instantaneous luminosity:

$$
\xi(t)= \begin{cases}4.15 & \text { for } t<\tau_{a} \\ \exp \left[\frac{\tau_{a}-t}{\tau_{c}}\right] & \text { for } t>\tau_{a}\end{cases}
$$

Of course, the value of $\mathcal{E}_{a} / \tau_{a}$ (the luminosity during accretion) determines the numerical factor 4.15 , and the value of the luminosity in the beginning of the cooling phase $\mathcal{E}_{c} / \tau_{c}$ gives $\xi(t)=1$ just after $t=\tau_{a}$. A straightforward calculation gives the new values for the probability $P_{i}$ that an individual event is due to background:

\begin{tabular}{c||ccc|ccc|ccc|ccc} 
event & 1 & 2 & 3 & 4 & 5 & 6 & 7 & 8 & 9 & 10 & 11 & 12 \\
$P_{i}$ & $0 \%$ & $0 \%$ & $41 \%$ & $9 \%$ & $1 \%$ & $94 \%$ & $0 \%$ & $0 \%$ & $0 \%$ & $67 \%$ & $2 \%$ & $67 \%$
\end{tabular}

(compare with Sect. 2.2.2). The most striking consequences of the new hypothesis is the increased probability that the event number 4 is due to supernova signal, whereas the late events have a higher chance to be caused by background. Due to these features, the expectations on the number of background events, based on the energy distribution, are not radically modified:

\begin{tabular}{c||ccccc} 
\# of bkgr. events $n$ & 1 & 2 & 3 & 4 & 5 \\
probability $\mathcal{P}_{n}$ & $7 \%$ & $28 \%$ & $41 \%$ & $20 \%$ & $2 \%$
\end{tabular}

\footnotetext{
${ }^{5}$ It is possible to somewhat improve the fit to the data using slighly different model parameters (e.g., a value of $\tau_{c}$ some times smaller than we assumed) but this would not change radically our conclusions.
} 
The probability that all events are signal is $0.36 \%$, and we can exclude at $10 \sigma$ that all events are due to background. However, there is an important difference with what we found based on the results of section 2.2. The events 3,4 (10) that are located close to the border of the detector have a smaller (larger) chance to be due to background, and this has an impact on global analyses. Using as a reference analysis $\mathrm{C} 2$ described above, we find the following results, that can be compared with those in section 3.1.1;

- The probability that no background events occurred becomes $0.9 \%$. This is small is absolute terms, but larger than estimated above using only the energy distribution.

- The probability that a certain number of background events occurred is:

\begin{tabular}{c||ccccc} 
\# of bkgr. events $n$ & 1 & 2 & 3 & 4 & 5 \\
probability $\mathcal{P}_{n}$ & $11 \%$ & $36 \%$ & $36 \%$ & $14 \%$ & $2 \%$
\end{tabular}

that is rather similar to the values calculated just above, based on the expected energy distribution only.

- The individual cases that occurred with probability above $5 \%$ are:

\begin{tabular}{c||c|c|c|c|c|c} 
background events & 6,10 & $3,6,10$ & $6,10,12$ & 6 & 3,6 & $3,6,10,12$ \\
probability $\mathcal{P}_{n}$ & $18.9 \%$ & $12.0 \%$ & $9.5 \%$ & $7.9 \%$ & $7.0 \%$ & $5.7 \%$ \\
\hline volumetric SCVM & $7.2 \%$ & $18.0 \%$ & $3.3 \%$ & $2.4 \%$ & $6.5 \%$ & $9.9 \%$ \\
angular SCVM & $3.3 \%$ & $1.2 \%$ & $1.8 \%$ & $8.4 \%$ & $4.0 \%$ & $5.2 \%$
\end{tabular}

where we give also the GOF of the volume and of the angular distributions in the last two lines. Note that these 6 cases alone cover $61 \%$ of the 4096 possibilities.

- The event number 6 is due to background in $90 \%$ of the cases.

In summary, the existence of a accretion phase brings a new twist in the analysis of the backgrounds, and leads to somewhat different conclusions especially regarding the interpretation of the event number 4. However, the new analysis still leads us to expect that some low energy events among the events number 3,6,10,12 could be attributed to background.

\subsubsection{Again on the interpretation of low energy events}

In view of the discussion of the previous section, the interpretation proposed in section 3.1 .2 does not need a real revision, except, possibly, replacing the event 4 with the event 12 in the role of candidate background event.

However, the wider theoretical context also allows us to take a new point of view, or in other words, it is possible to formulate another way out from the 'problem' of low energy events. In fact, one could argue that the events occurred during the non-thermal phase (accretion) should not be compared with the ones occurred later. In particular, the average energy of the first four $\mathrm{KII}$ events is $12.6 \pm 2.4 \mathrm{MeV}$, that is pretty much lower than the one of the next seven events (the event number 6 being attributed to background), namely $17.1 \pm 3.3 \mathrm{MeV}$. Of course this is due to the presence of a few low energy events among the first events (the events 3 and 4). But while in section 3.1 .2 we regarded them as as possible background events, here we are lead to suggest 
that they (or at least some of them) are due to a peculiar phase of neutrino emission, that in principle could be characterized by a lower average energy than during thermal phase. In short, the fact that KII measured several low energy events can be at least partly attributed to the fact that some of these events occurred during accretion. In order to have an acceptable volumetric distribution, it is sufficient to assign only one additional event between 3 and 10 (along with event 6) to background.

\section{Discussion}

We have shown in Sect. 3 that the agreement between the observed and expected average energy of supernova neutrinos improves significantly assuming that 2-4 of the low energy events in KII are due to background, and that this interpretation is supported by the volumetric distribution of the events. A few of these low energy events could also belong to a peculiar phase of neutrino emission ("accretion"), contributing to solve the 'problem'. In other words, we believe that the null hypothesis (i.e., the minimal model) is not significantly challenged by the excess of low energy events observed by Kamiokande-II (or: we have enough freedom to evade the conclusion that there is a problem with the conventional expectations). Here, we would like to offer various remarks on this conclusion.

New tools used. A new crucial information that permitted us to reach new conclusions is the analysis of the spatial distribution of the events (Sect. 2.1). The validity of the hypothesis on the background that we formulated in Sects. 3.1.2 and 3.2.3 rests also, to a certain extent, on the assumption we made on the signal of supernova neutrinos described in the beginning of Sects. 2 and 3.2.1. In our view, these should be considered a conservative and reasonable assumptions on the signal. They are consistent with the IMB observations that are basically background free. Furthermore, the conclusions we reach remain valid under small modifications of the model for supernova neutrinos, and do not contradict (but rather strengthen) what we obtain following Kamiokande-II and setting the energy cut at $7.5 \mathrm{MeV}$ (Sect. 2.3).

Peculiarity of the approach. The simple but crucial observable that we have used to draw our conclusions is the average value of the visible energy, see in particular Sects. 3.1.2 and 3.2.3. A detailed comparison of the energy distributions in figure 2 would suggest two anomalies, namely: a) a defect of high energy events around $20-40 \mathrm{MeV}$ and b) an excess of events around $10 \mathrm{MeV}$. While we argued that the low energy anomaly could be due to background events (or to the fact that several events occurred during accretion) we are unable to explain the high energy anomaly if not resorting to a fluctuation of the data. However, 'global' observables are safer against the effects of the fluctuations; thus, in view of the low number of collected events we emphasized the comparison of average visible energies rather than the comparison of energy distributions. In this respect, our formulation of the 'problem' of low energy events, or equivalently our approach to KII dataset, are more conservative and distinct from the ones of other recent analyses such as [25] and [26], and closer to the one of [23] (see figure 1 there). A quantitative statement is in the first paragraph of Sect. 2.2.1.

Leftover possibilities and/or alternative (but not exclusive) keys of interpretation. It is not impossible that other reasonable effects contribute to explain the apparent excess of low energy 
events (and/or defect of high energy events) in KII dataset, for instance:

(i) The measured energies should not be thought of as Gaussians (as suggested by the reported data). If we use errors that scale as $\sqrt{E}$ [19] rather than being constant as assumed in the rest of the paper (equation 5) we see a modest improvement of the agreement in the region dominated by the background (i.e., low energies).

(ii) There is one event due to elastic scattering that degrades the visible energy as allowed by the selected theoretical model of emission [23]. This option is interesting since when assigning events to background, the angular distribution does not improve much (see e.g., figure 3).

(iii) The neutrino flux in the thermal phase deviates slightly from expectations, say, in the direction suggested by [25] but possibly not that radically (in our evaluations, the change is not large-see Sect. 3.1.1).

(iv) Some observed event is really due to supernova neutrinos, but it suffers of a very poor energy reconstruction (though, we do not have the necessary information to elaborate on this possibility).

$(v)$ Some observed events are due to supernova neutrinos, not necessarily $\bar{\nu}_{e}$, interacting with the walls of the detector (a similar possibility was originally noticed for non-standard collapses [16, but was shown to yield a relevant amount of events in existing detectors also for a standard collapse [17).

(vi) The time structure of the signal is significantly different from what we know, e.g., the phase of accretion is much more luminous (see Sect. 3.2.1).

While most possibilities are largely within conventional expectations, some of them go beyond the minimal model that we formulated. This is true for the last two possibilities listed just above, that are however tightly connected with the most unknown aspects regarding the explosion, and (if proven to be viable) could offer us new insights on what happened during SN1987A neutrino burst.

Possible future developments. With more information, more detailed analyses of the KII data could be possible. In fact, one should analyze the energy and the spatial distribution at the same time, assuming that the observations are due to several phenomena; a signal of high energy due to neutrino interactions with free protons, with electrons and with oxygen nuclei, uniformly distributed in the detector (and possibly, also a hypothetical component of the signal concentrated on the wall of the detector); a low energy background component, similarly distributed, with (in principle) known intensity; a more energetic background component, concentrated in the wall of the detector, also known (again, in principle). More discussion of these needs is in App. A and App. B. Another interesting thing would be the calculation of the best fit for the supernova neutrinos parameters, keeping into account the presence of background in KII, with peculiar (and in principle known) spatial and energy distributions. For lack of information-as discussed in the beginning of Sect. 2, compare also with the discussion of equation 3.22 in [21]-we have been forced to analyze the energy and the spatial distributions separately, simply distinguishing in each analysis between a signal and a background component. Perhaps, more detailed analyses like these will be carried out in the future; in the meantime and view of what we learned here, we are lead to expect as a plausible outcome that the event 6 should be attributed to background because of its low energy, and some events among 3, 4, 10 (resp., 10, 12) should be attributed to background mostly because of their spatial distribution (resp., of their relatively late occurrence).

We wish to close recalling the warning of E N Alexeev: "it is possible that some of the low energy events carry an important message, still to be understood". We believe that these are 


\begin{tabular}{c|cccccccccccccccc}
$i$ & 1 & 2 & 3 & 4 & 5 & 6 & 7 & 8 & 9 & 10 & 11 & 12 & 13 & 14 & 15 & 16 \\
\hline$B_{i}^{L L}$ & $1.6_{5}$ & $1.9_{3}$ & $2.9_{2}$ & $1.2_{2}$ & $2.1_{3}$ & $3.7_{2}$ & $4.5_{5}$ & $8.2_{5}$ & $1.5_{5}$ & $1.5_{2}$ & $1.9_{3}$ & $1.6_{2}$ & $3.8_{2}$ & $2.9_{2}$ & $2.8_{2}$ & $3.8_{2}$ \\
$B_{i}$ & $1.0_{5}$ & $5.4_{4}$ & $3.1_{2}$ & $8.5_{3}$ & $5.3_{4}$ & $7.1_{2}$ & $5.0_{6}$ & $1.0_{5}$ & $1.0_{5}$ & $1.8_{2}$ & $4.0_{4}$ & $1.4_{2}$ & $7.3_{2}$ & $5.2_{2}$ & $1.8_{2}$ & $7.3_{2}$
\end{tabular}

Table 4: $1^{\text {st }}$ line, the progressive event number; next, the estimated background rate in $\mathrm{Hz} / \mathrm{MeV}$ according to the procedure of the 'convolution integrals' followed by LL (2 $2^{\text {nd }}$ line) and the procedure we describe in the text ( $3^{\text {rd }}$ line). A subscript indicates the exponent: e.g., $3_{2}$ means $3 \cdot 10^{-2}$.

wise words and we subscribe them; as we saw, the message could be the existence of a luminous phase of neutrino emission (Sect. 3.2). However, we also believe that it is important to check how close (or how far) we can go to describe the data using only conventional hypotheses on supernova neutrinos, and this is what we tried to do in this paper. Certainly, the handful of events collected from SN1987A was enough for the first observation of supernova neutrinos (the quantitative statement on the significance of KII observation is in Sect. 2.2.2), but as we showed with our analysis, the possibility that some of these events are not due to $\bar{\nu}_{e} p \rightarrow n e^{+}$should suggest caution whenever we attempt to infer the characteristics of SN1987A neutrino emission from the observations.

\section{Acknowledgments}

We thank for discussions F Burgio, G L Fogli, W Fulgione, P L Ghia, P Lipari, E Lisi, A Mal'gin, D K Nadyozhin, O G Ryazhskaya, M Selvi, A Yu Smirnov, A Strumia, F L Villante and the anonymous Referee who offered us the occasion to clarify and elaborate the results of this work.

\section{A The assumed background}

We interpret the curve $B(E)$ presented in fig. 2 of [21] as the measured background rate for the estimated event energy. We tested this interpretation by constructing a histogram from fig. 4 in [5] and using a linear conversion factor between $N_{\text {hit }}$ and $E$, fixed by the correspondence between known values: $N_{\text {hit }}=20 \Leftrightarrow 7.5 \mathrm{MeV}$ and/or $N_{\text {hit }}=26 \Leftrightarrow 10 \mathrm{MeV}$. Within limited statistics, the histogram coincides with the curve given by Lamb and Loredo (LL). Note that our interpretation agrees with the one of LL [21, who state "KII and Baksan provided us with measurements of $B(E)$ " and later describe their fig. 2 as "background rate measurements". Now, since the measured background already includes the effects of the fluctuations in the energy measurements ('smearing'), we estimate the background rate for the individual event using the central value of the measured energy: $B_{i}=B\left(E_{i}\right)$, see table 4. A different prescription was followed in [21], namely, $B_{i}^{L L}$ was evaluated by taking the convolution integral of the curve $B(E)$ with the Gaussian energy distribution of the individual events; the values from table III of [21] are given for comparison. In our understanding, such a prescription leads to double counting the effect of 'smearing', and thus (1) to underestimate the $B_{i}$ for the events with energy close to the background peak, and (2) to overestimate those in the tails of the $B(E)$ distribution 6 The

\footnotetext{
${ }^{6}$ We believe that the procedure of taking the convolution integrals should be applied to the true energy distribution of the background, namely, the distribution that does not include the fluctuations in the energy
} 
new values of $B_{i}$ have been obtained as follows: below $10 \mathrm{MeV}$, we obtain accurate data from the plot of LL, whereas, above, this is not of much use; above $9 \mathrm{MeV}$, however, we can use the background curves denoted as STEP1 and STEP2 shown in fig.3 of [6] for the purpose of solar neutrino studies, with the conversion between energy and $N_{\text {hit }}$ given there (STEP1 agrees with the curve of LL, where comparable). Below $7 \mathrm{MeV}$, the new procedure leads to higher $B_{i}$ (ev.s 6, 13-16); from 7.5 to about $9 \mathrm{MeV}$, there is no significant difference with the procedure of LL (ev.s 3,10,12); between 9 ad $17 \mathrm{MeV}$, the background curves in [6] suggest a lower $B_{i}$ (ev.s $2,4,5,11)$; above-in the most uncertain but safest from the background point of view, and also less crucial region for parameter estimation-we assume a lower background rate than assumed by LL (ev.s 1, 7-9). More precise values could be provided by the experimental collaboration using $B(E, \vec{x})$-not the average value $B(E)$-thus describing the role of the position of the individual events (similarly for detection efficiencies). It should be noted that the changes in the values of the $B_{i}$ that we propose are not crucial for the analysis; their main effect is just to increase the $a$ priori probability that the event number 6 is due to background, as in the first and most popular interpretation [1].

\section{B The assumption of uniform volumetric distribution}

We analyze critically the hypothesis that the events from the signal are uniformly distributed. In the relevant publications [1, 5, 7, 6] we were not able to find warnings against important systematics of this type, but we tentatively identified three such physical effects that could affect the observed signal events:

Geometry: The events occurring close to the border and propagating toward the closer wall could be missed, since the Čerenkov light could pass through the phototubes without being recorded. In our understanding, this is the main reason why the detection efficiency of Kamiokande-II never reaches $100 \%$, even for the higher energy events, but is at most $92 \%$. In order to test this hypothesis, we considered the external part of the detector that has a distance from the phototubes smaller than their typical distance of 1 meter. Since this part has a volume of about $800 \mathrm{~m}^{3}$, we reproduce the $92 \%$ efficiency if $\sim 20 \%$ of the events are lost in this way, that is a reasonable figure being comfortably smaller than $40 \%$. Again, this effect leads us to expect that the loss of signal events is more significant for the events produced close to the border, especially for $f \geq 0.6$, that corresponds to the external part of the detector considered just above. We attempt to quantify the size of this geometrical effect by the function $G[f]=1-(f-0.4)^{2} \theta[f-0.4]$, where we used a quadratic function to describe a smooth behavior $(\theta[x]$ is the Heaviside function). This means that $\sim 8 \%$ of the total number of events are systematically lost close to the border, and that even at $f=1$, the loss is smaller than $40 \%$.

Light attenuation: The light from the low energy events can be lost because of light attenuation, where $\lambda \sim 50 \mathrm{~m}$ (in [5], we read that $\lambda$ "exceeds $50 \mathrm{~m}$ at any time"). Let us evaluate the average attenuation coefficient for Kamiokande-II detector, as a function of the volumetric coordinate $f$. Considering the simplified case of propagation in straight rays, we find the differential distribution in the variable $f$ by averaging over the other coordinates that specify the position of the event

measurement. The convolution integral would then amount to include the effect of the fluctuations on individual basis, and could lead to a more accurate background evaluation. 
and the direction of emission:

$$
\frac{d A}{d f}=\int_{0}^{1} d c \int_{0}^{2 \pi} d \phi \int_{0}^{1} d u \frac{u A\left[u f^{1 / 3}, f^{1 / 3}, c, \phi\right]+A\left[f^{1 / 3}, u f^{1 / 3}, c, \phi\right]}{3 \pi}
$$

where $A[r, h, c, \phi]=\left(e^{-D_{\text {in }} / \lambda}+e^{-D_{\text {out }} / \lambda}\right) / 2$ is the average attenuation for a given direction of observation, $D_{\text {in }}$ and $D_{\text {out }}$ are the two distances from the walls of the detector (see also table 11), and we measure the radial coordinate $r$ in units of $R$ and the height $h$ in units of $H / 2$. A straightforward calculation gives the approximation for $d A / d f \approx 0.858+0.068 f$, that means that the events produced in the center suffer an attenuation effect that is $\sim 10 \%$ larger. The systematic effect due to light attenuation depends on the energy. If we assume that the signal is uniformly distributed in energy, we are lead to expect that the loss of low energy events from the center is more significant.

Spectrum: However, the previous point does not take into account the real spectrum of the signal. In order to demonstrate that this is also relevant, it is enough to consider a simple one dimensional situation, when the light propagates just in one direction or the opposite one. Let us compare what happens to the events produced in the middle $(f \sim 0)$ and in the border of the detector $(f \sim 1)$. In the first case, the light attention effect is $e^{-L / 2 \lambda} \approx 0.87$, where $L=14 \mathrm{~m}$ is the linear size of the detector; in the second case, this depends on whether the event propagates toward the closer border (no attenuation) or toward the opposite border, when we have $e^{-L / \lambda} \approx 0.76$. In the first case, we lose all the events between $N_{\min }$ and $1.15 N_{\min }$, where $N_{\min }$ is the minimal number of photons that gives a trigger; in the second one, we lose half of the events between $N_{\min }$ and $1.3 N_{\min }$ (where $e^{L / \lambda} \approx 1.3$ ). If the signal is uniformly distributed in energy, we see that at $\mathcal{O}(L / \lambda)$ the effect is identical in the two cases; thus, the effect described by eq. 13 is purely three dimensional. But if the distribution of the signal increases with the energy, as we expect, the loss of signal events is more significant for the events produced close to the border. In fact, the events that fall below the detection threshold in the first case (events produced close to $f \sim 0$ ) are those that belong to the least populated part of the spectrum. E.g., if we assume that the energy spectrum of the signal rises linearly from zero, the total number of remaining events in the region $N_{\min }-1.3 N_{\min }$ is $50 \%$ larger in the first case. This last effect is presumably the less relevant one.

In summary, various effects can change the distribution of the detected signal events: the second effect increases a bit the number of expected supernova neutrino events close to the border, the other two diminish it. It is not possible to estimate precisely the systematics deviations from uniformity without a detailed simulation of the detector response that takes into account the energy distribution of the signal, the role of energy fluctuations, etc. However, if we account for the two systematics effects that work in opposite directions simply by multipling $G[f]$ by $d A / d f[f]$, we see that the geometrical effect is the most important one. This is the reason why we claim that the GOF with a uniform volume distribution underestimates a possible problem with events close to the border: in fact, when we apply the SCVM test to the 12 events seen by Kamiokande-II we get a GOF of $3.4 \%$ rather than the value that we obtain with uniform distribution, $4.6 \%$ (see table 22). Similarly, when we discard event 6 , the value of the GOF becomes $1.8 \%$ rather than $2.4 \%$ (again in table 21). As we see, the changes due to a more accurate modeling of the detector response are not expected to be very large, and are in any case smaller than the changes due to the use of alternative statistical tests (see next Appendix). 


\section{The Anderson-Darling test}

The Anderson-Darling (AD) test [27] is used to test if a sample of data comes from a specific distribution. It is a modification of the Kolmogorov-Smirnov test and gives more weight to the boundary region than the Smirnov-Cramèr-Von Mises test.

Consider a set of data $x_{1}, x_{2}, \ldots x_{n}$, arranged in increasing order. Given the expected cumulative distribution function $F[x]$, we calculate the values in the points $x_{j}$ :

$$
F_{j}=F\left[x_{j}\right]
$$

Next, consider the empirical cumulative distribution function $F_{n}[x]$, that counts the number of events below $x$; thus, $F_{n}[x]=j / n$ for $F_{j} \leq u<F_{j+1}$, where we set $F_{0}=0$ and $F_{n+1}=1$. The quantitative indicator of the likelihood of the given data proposed by Anderson and Darling is:

$$
\left.W^{2} \equiv n \int_{0}^{1}\left(F_{n}[x]-u\right)^{2} \psi[u] d u\right|_{F[x]=u}
$$

where $\psi$ is a positive function on the interval $(0,1)$. The case when $\psi=1$ gives the well-known Smirnov-Cramèr-Von Mises test, that somehow resembles the $\chi^{2}$ statistics:

$$
W_{\mathrm{scvm}}^{2}=\frac{1}{12 n}+\sum_{j=1}^{n}\left(F_{j}-\frac{j-1 / 2}{n}\right)^{2}
$$

Let us pass to the alternative possibilities. The traditional (two sided) version of the AndersonDarling test statistic is obtained setting $\psi[u]=1 /(u(1-u))$, that gives:

$$
W_{\text {traditional }}^{2}=-n-\sum_{j=1}^{n} \frac{2 j-1}{n} \log \left[F_{j}\left(1-F_{n+1-j}\right)\right]
$$

This provides a particular sensitivity close to the points $u=0$ and $u=1$. A trivial modification consists in setting $\psi[u]=1 /(1-u)$ : in this way, the test becomes particularly sensitive close to $u=1$. The modified (or one sided) Anderson-Darling test statistic can be written as:

$$
W_{\text {modified }}^{2}=\frac{n}{2}-2 \sum_{j=1}^{n} F_{j}-\sum_{j=1}^{n} \frac{2 j-1}{n} \log \left[1-F_{n+1-j}\right]
$$

Since $1 /(u(1-u))=1 / u+1 /(1-u)$, equation 17 can be recovered by summing a third test statistic when we replace $\psi[u]=1 / u$, that would be suited to test for features close to $u=0$ (the practical formula for this third test statistic is obtained replacing $F_{j} \rightarrow 1-F_{n+1-j}$ in equation [18).

A null-hypothesis that we want to test is whether a pre-selected subset of the KII data is uniformly distributed in the detector, as expected if they are due to supernova neutrino events. Thus, in our case the number of data is $n \leq 12$, the coordinate $x$ is what is called $f$ in Sect. 2.1, and the cumulative distribution function is simply $F[x]=x$. The AD tests of Eqs. [17 and [18 are of particular interest since the points close to $f=1$ are in the border of the volume used in the analysis, and, there, it is not possible to exclude a priori an effect of contamination from the background. 
Given the test statistics, we calculate the value $W_{*}^{2}$ for the subset of KII data in which we are interested. How often this value results from a uniform distribution? This can be obtained with a straightforward procedure: 1) we extract randomly $n$ data from a uniform distribution for $N$ times ( $N=10^{8}$ in our case); 2) we calculate the value of $W^{2}$ for each data set; 3 ) finally, we test the frequency of the condition $W^{2}>W_{*}^{2}$, namely, the number of times $N_{*}$ that this condition is satisfied over the number of extractions $N$.

E.g., we infer from the test of equation 17 that when all 12 events (resp., all except event 6) are supposed to be due to supernova neutrinos, we get $W_{*}^{2}=4.95$ (resp., $W_{*}^{2}=5.80$ ). A value this high can occur as a result of a statistical fluctuations, but only in $0.32 \%$ (resp., 0.13 $\%$ ) of the cases; this means that we can reject the hypothesis that the 12 (resp., 11) events are uniformly distributed at the $2.9 \sigma$ (resp., $3.2 \sigma$ ) significance level. Similarly, we infer from the test of equation 18 that the case when all events (resp., all except event 6) are assumed to be signal can be rejected at the $3.5 \sigma$ (resp., $3.8 \sigma$ ) significance level.

In principle, it is possible to object that the situations when different statistical tests give very different outcomes should be regarded as dubious and that the statistical criterion would be better selected a priori rather than a posteriori; these are the reasons why in this paper we do not use exclusively the (more powerful) AD test, but also the (more conventional) SCVM test. 


\section{References}

The references are listed in order of time within 6 topics: SN1987A data [1, 2, 3, 4, other relevant information on Kamiokande-II [5, 6, 7]; theoretical works on supernova neutrinos [8, 9, 10, 11, 13, 12, 14, 15, 16, 17, 18; (an incomplete list of) analyses of SN1987A data [19, 20, 21, 22, 23, 24, 25, 26]; statistics 27, 28, 29; talks at three recent conferences on SN1987A 30].

[1] K Hirata et al [Kamiokande-II Collaboration], Phys. Rev. Lett. 58 (1987) 1490.

[2] R M Bionta et al., Phys. Rev. Lett. 58 (1987) 1494.

[3] E N Alekseev, L N Alekseeva, I V Krivosheina and V I Volchenko, Phys. Lett. B 205, 209 (1988).

[4] M Aglietta et al., Europhys. Lett. 3 (1987) 1315 .

[5] K S Hirata et al., Phys. Rev. D 38 (1988) 448.

[6] K S Hirata et al., in "The Standard model: supernova 1987A" proceedings of leptonic session of XXII ${ }^{\text {nd }}$ Rencontre de Moriond, Les Arcs, March 1988 edited by J. Tran Thanh Van, p. 689.

[7] K S Hirata, "Search for supernova neutrinos at Kamiokande-II", Tokyo Univ. Ph.D. Thesis, ICRR-239-91-08.

[8] D K Nadyozhin, Astrophys. Space Sci. 53 (1978) 131.

[9] J R Wilson in Numerical Astrophysics, eds. J Centrella, J LeBlanc, R L Bowers, page 422 (Jones and Bartlett, Boston, 1985).

[10] H A Bethe and J R Wilson, Astrophys. J. 295 (1985) 14.

[11] J M Lattimer, A Yahil, Astrophys. J. 340 (1989) 426.

[12] J N Bahcall, cap 15 of Neutrino astrophysics, Cambridge University Press, 1989.

[13] D K Nadyozhin in Supernovae, eds. S A Bludman, R Mochkovitch, J Zinn-Justin, page 301 (Les Houches session LIV, 1990, North Holland \& Elsevier, 1994).

[14] M T Keil, G G Raffelt and H T Janka, Astrophys. J. $\mathbf{5 9 0}$ (2003) 971 arXiv:astro-ph/0208035.
[15] A Strumia and F Vissani, Phys. Lett. B 564 (2003) 42 arXiv:astro-ph/0302055.

[16] V S Imshennik and O G Ryazhskaya, Astron. Lett. 30 (2004) 14.

[17] N Y Agafonova et al., Astropart. Phys. 27 (2007) 254 arXiv:hep-ph/0609305.

[18] H Th Janka, K Langanke, A Marek, G Martínez-Pinedo, B Müller, submitted to the Bethe Centennial Volume of Physics Reports astro-ph/0612072].

[19] B Jegerlehner, F Neubig and G Raffelt, Phys. Rev. D $\mathbf{5 4}$ (1996) 1194 arXiv:astro-ph/9601111.

[20] A S Mal'gin, Nuovo Cim. C 21 (1998) 317.

[21] T J Loredo and D Q Lamb, Phys. Rev. D 65 (2002) 063002 arXiv:astro-ph/0107260.

[22] Y Totsuka, A Mann, S -B Kim as quoted in previous paper.

[23] M L Costantini, A Ianni and F Vissani, Phys. Rev. D 70 (2004) 043006 arXiv:astro-ph/0403436.

[24] D K Nadyozhin and V S Imshennik, Int. J. Mod. Phys. A 20 (2005) 6597.

[25] A Mirizzi and G G Raffelt, Phys. Rev. D 72 (2005) 063001 arXiv:astro-ph/0508612.

[26] C Lunardini, Astropart. Phys. 26 (2006) 190 arXiv:astro-ph/0509233.

[27] M A Stephens, Journal of the American Statistical Association 69 (1974) 730.

[28] S Csorgo, J J Faraway, Journal of the Royal Statistical Society B 58 (1996) 221.

[29] T W Anderson, D A Darling, American Statistical Association Journal 49 (1954) 765.

[30] Web sites the conferences held in Moscow: http://lvd.ras.ru/SN1987A/en/materials/index.php; Hawaii, http://sn1987a-20th.physics.uci.edu/Program1.htm; Venice, http://neutrino.pd.infn.it/conference2007/talks.html. 